\title{
The interstellar extinction to V4334 Sgr (Sakurai's object)
}

\author{
A. Evans ${ }^{1}$, T. R. Geballe ${ }^{2}$, B. Smalley ${ }^{1}$, V. H. Tyne ${ }^{1}$, and S. P. S. Eyres ${ }^{3}$ \\ 1 Department of Physics, Keele University, Keele, Staffordshire, ST5 5BG, UK \\ 2 Gemini Observatory, 670 N. A'ohoku Place, Hilo, HI 96720, USA \\ ${ }^{3}$ Centre for Astrophysics, University of Central Lancashire, Preston, Lancashire, PR1 2HE, UK
}

Received 8 August 2002 / Accepted 26 August 2002

\begin{abstract}
We present an 8-13 $\mu \mathrm{m}$ spectrum of V4334 Sgr (Sakurai's object), which shows clear evidence for the presence of a silicate absorption feature at $9.7 \mu \mathrm{m}$. We argue that the feature is interstellar in origin, and on this basis we determine the interstellar extinction to V4334 Sgr to be $E(B-V)=0.66 \pm 0.12$. This is the only determination of the extinction to V4334 Sgr to date that uses direct observation of the intervening material; as the reddening using this method is refined it will be of importance in determining the distance of V4334 Sgr, and hence in modelling its evolution.
\end{abstract}

Key words. stars: evolution - ISM: dust, extinction - infrared: ISM - stars: individual: V4334 Sgr - stars: variables: general

\section{Introduction}

Sakurai's Object (V4334 Sgr) has been the subject of considerable interest over the past 5 years (see Evans \& Smalley 2002). It is undergoing a late thermal pulse, returning from the white dwarf cooling track and retracing its evolution to become a "born-again" red giant. Recent observations (Kerber et al. 2002) suggest that Sakurai's object may now be heating up, and retracing its post-AGB evolution.

Modelling this poorly-understood (or indeed any) phase of stellar evolution requires reliable values of the interstellar extinction and distance. There have been various determinations of the interstellar extinction to V4334 Sgr. These are based on (i) the $\mathrm{H} \alpha / \mathrm{H} \beta$ ratio in the planetary nebula (PN), (ii) the freefree flux in the radio PN compared with the $\mathrm{H} \beta$ flux, (iii) comparison of the observed stellar spectrum with a suitable model atmosphere and (iv) the dependence of reddening on distance to V4334 Sgr (see Kimeswenger 2002 for a detailed review). The reddening-distance method as applied by Kimeswenger \& Kerber (1998) to V4334 Sgr has recently been refined and extended by Pirzkal et al. (2001). These methods produce values of $E(B-V)$ in the range 0.65 to 0.85 , and Kimeswenger (2002) recommends $E(B-V)=0.75 \pm 0.05$.

We present here an 8-13 $\mu \mathrm{m}$ spectrum of V4334 Sgr, which provides a further, independent, determination of interstellar extinction.

\section{Observations}

The spectrum was obtained on UT 2002 July 15 with the facility instrument Michelle (Glasse et al. 1997) on the United Kingdom Infrared Telescope (UKIRT). The low resolution

Send offprint requests to: A. Evans, e-mail: ae@astro.keele.ac.uk grating was used with a 2 pixel (0.76 arcsec) wide slit, giving a resolving power $\lambda / \Delta \lambda \simeq 200$. Sakurai's object was observed in the standard stare/nod mode, at an average airmass of 1.28. The bright star HR 6378 (assumed to have $N=2.25$ and $T_{\text {eff }}=8800 \mathrm{~K}$ ) was observed in the same way at a mean airmass of 1.24, for the purposes of flux calibration and removing telluric features. Telluric absorption features provided the wavelength calibration, which is accurate to $0.02 \mu \mathrm{m}$.

The reduced $8-13 \mu \mathrm{m}$ spectrum of V4334 Sgr is shown in Fig. 1a. An apparent broad absorption feature, centered between 9.5 and $10.0 \mu \mathrm{m}$, resembles the absorption profile of astronomical silicates. The spectrum is noisy near the maximum absorption, which coincides with the strongest part of the deep telluric ozone absorption near $9.6 \mu \mathrm{m}$. The airmass mismatch would tend to artificially deepen the feature in the reduced spectrum, but the effect is small compared to the observed feature, because the mismatch was small and because many of the individual ozone lines are saturated. We have made no attempt to correct for the mismatch.

\section{Discussion}

\subsection{The extinction in the silicate feature}

A spectrum in the $8-13 \mu \mathrm{m}$ window, obtained on 1998 October 1, was presented by Lynch et al. (2002), who noted the absence of a $9.7 \mu \mathrm{m}$ silicate feature and of hydrocarbon features, but suspected a broad, weak emission feature between 8 and $9 \mu \mathrm{m}$ relative to a $1000 \mathrm{~K}$ blackbody.

However in our data the silicate feature, in absorption, is clearly visible (see Fig. 1a); we do not confirm the feature noted by Lynch et al. (2002). Our spectrum might be interpreted as containing an $8-9.5 \mu \mathrm{m}$ emission feature of unknown origin, 
A. Evans et al.: The interstellar extinction to V4334 Sgr (Sakurai's object)
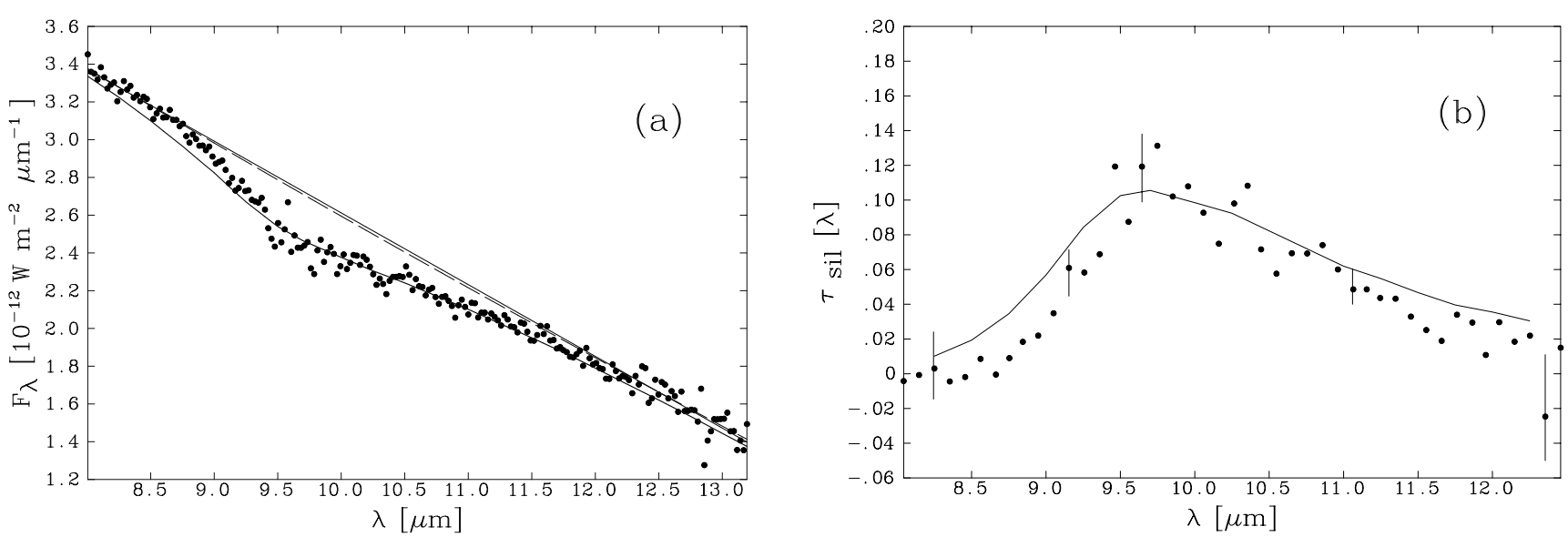

Fig. 1. a) The $8-13 \mu \mathrm{m}$ spectrum of V4334 Sgr. Solid line is linear continuum, broken line quadratic continuum, curve is continuum with silicate feature superimposed; see text for details. b) The profile of the silicate absorption feature, based on linear continuum; data have been binned into $0.1 \mu \mathrm{m}$ bins. The curve is the interstellar silicate feature as discussed in text; the peak of the curve is normalized at $\tau=0.105$ and $\lambda=9.7 \mu \mathrm{m}$. Error bars give typical spread of data within bins at selected wavelengths.

but doing so would either imply a very peculiar continuum shape longward of $9.5 \mu \mathrm{m}$, or a second broad and unknown emission feature longward of $9.5 \mu \mathrm{m}$. It is more logical to interpret the spectral structure as a well-known absorption feature. The emission feature noted by Lynch et al. either may have been an artefact of data reduction (which Lynch et al. state is unlikely), or its carrier is no longer present, or (in our opinion the most likely explanation) the $1000 \mathrm{~K}$ blackbody assumed by Lynch et al. was not a good approximation to the dust continuum (see below).

Tyne et al. (2002) have modelled the dust emission from V4334 Sgr and in principle we could use similar modelling to define the continuum for our $8-13 \mu \mathrm{m}$ observation. However at present we have no other constraints on the dust emission and, for the purpose of extracting the profile of the silicate feature, we attempt two alternative fits to the dust continuum over the wavelength ranges $8-8.7 \mu \mathrm{m}$ and $12.1-12.8 \mu \mathrm{m}$ (i.e. avoiding the $9.7 \mu \mathrm{m}$ feature). We shall see below that the calibration uncertainties exceed the uncertainty in placing the continuum. We consider a linear function of the form

$F_{\lambda}=A+B \lambda$,

and a quadratic of the form

$F_{\lambda}=A^{\prime}+B^{\prime} \lambda+C^{\prime} \lambda^{2}$,

where $F_{\lambda}$ is the flux in units of $10^{-12} \mathrm{~W} \mathrm{~m}^{-2} \mu \mathrm{m}^{-1}$. The values of the coefficients $A, B$ etc. are given in Table 1 ; these functions are included in Fig. 1a. We note that the coefficient of the quadratic term $\left(C^{\prime}\right)$ is not significantly different from zero and henceforth we consider only the linear continuum.

The observed spectrum and fitted linear continuum have been ratioed to determine the optical depth $\tau_{\text {sil }}(\lambda)$ in the silicate feature in the wavelength range $8-12.5 \mu \mathrm{m}$ (see Fig. 1b). As noted, the uncertainty in the flux calibration far exceeds the uncertainty in the placement of the continuum, and we determine from Fig. 1b that the optical depth at the peak of the silicate feature is

$\tau_{9.7}=0.105 \pm 0.005$.
Table 1. Coefficients in least squares fit to $8-13 \mu \mathrm{m}$ continuum.

\begin{tabular}{rcrcr}
\hline \hline \multirow{2}{*}{ Linear } & \multicolumn{1}{c}{$\longleftarrow$} & Quadratic & $\longrightarrow$ \\
\hline \multicolumn{1}{c}{$A$} & $B$ & \multicolumn{1}{c}{$A^{\prime}$} & $B^{\prime}$ & \multicolumn{1}{c}{$C^{\prime}$} \\
\hline 6.4122 & -0.3800 & 6.8424 & -0.4663 & 0.00415 \\
\pm 0.0495 & \pm 0.0047 & \pm 1.1790 & \pm 0.2359 & \pm 0.01135 \\
\hline
\end{tabular}

\subsection{Circumstellar silicate?}

V4334 Sgr is currently enveloped in a carbon-rich dust shell that is optically thick in the visual and near-infrared (Tyne et al. 2002). Before it was obliterated by the dust shell, the underlying star was also determined to be carbon-rich (Asplund et al. 1997). Under these circumstances the circumstellar dust is not expected to contain (oxygen-rich) silicates.

However as V4334 Sgr is known to be a born-again object it is possible that the silicate absorption may arise in a fossil shell ejected during an earlier phase of stellar evolution, but we consider this unlikely. The silicate feature in V4334 Sgr is optically thin $\left(\tau_{9.7} \simeq 0.1\right)$, from which it is reasonable to conclude that the optical depth in the visual $\tau_{\mathrm{v}}<0.1$ for any circumstellar silicate. In general, theoretical studies of the spectral energy distributions of circumstellar silicates (e.g. Ivezić \& Elitzur 1997) suggest that the $9.7 \mu \mathrm{m}$ silicate feature appears in emission when $\tau_{\mathrm{v}} \lesssim 1$. Furthermore, where the $9.7 \mu \mathrm{m}$ silicate feature is seen in carbon stars (e.g. Yamamura et al. 2000; Molster et al. 2001) and arises from material ejected during an earlier, oxygen-rich, phase it is indeed usually seen in emission. We conclude that the evidence is against the silicate feature in Sakurai's object being associated with an earlier evolutionary phase, and we proceed on the assumption that the silicate feature in V4334 Sgr has interstellar origin.

If the feature is indeed interstellar, it must have been present in the spectrum obtained by Lynch et al. (2002), although these authors noted its absence. However as we noted above, these authors may have been misled by their assumed $1000 \mathrm{~K}$ continuum; if instead the continuum is placed at the extreme ends of 
Table 2. Reddening to V4334 Sgr.

\begin{tabular}{lll}
\hline \hline$E(B-V)$ & \multicolumn{1}{c}{ Method } & \multicolumn{1}{c}{ Reference } \\
\hline 0.54 & Balmer decrement in PN & Duerbeck \& Benetti (1996) \\
0.79 & Balmer decrement in PN & Kerber et al. (2000) \\
$0.71 \pm 0.09$ & Case B H $\alpha / \mathrm{H} \beta$ ratio in PN & Pollacco (1999) \\
$0.76 \pm 0.20$ & Radio/H $\beta$ ratio in PN & Eyres et al. (1998), as modified by Kimeswenger (2002) \\
0.70 & Optical spectrum of star & Pavlenko et al. (2000) \\
$0.75 \pm 0.05$ & Weighted mean of several methods & Kimeswenger (2002) \\
$0.66 \pm 0.12$ & Interstellar silicate & This paper \\
\hline
\end{tabular}

their $10 \mu \mathrm{m}$ spectrum there is clearly present a broad absorption feature of comparable depth to the feature shown in Fig. 1a.

\subsection{The interstellar extinction and reddening}

For dust in the diffuse interstellar medium, the value of $\tau_{9.7}$ is well-known to correlate with the visual extinction, although the relationship is in general notoriously difficult to calibrate (cf. Mathis 1998). Mathis (1998) determines that $A_{\mathrm{v}} / \tau_{9.7} \simeq$ 19.3 , with an uncertainty of \pm 2.2 . This leads to

$A_{\mathrm{v}}=2.03 \pm 0.25$

and

$E(B-V)=0.66 \pm 0.12$

in the direction of V4334 Sgr; we have assumed a ratio of totalto-selective extinction of $3.05 \pm 0.05$ (Martin \& Whittet 1990). The largest source of uncertainty in our estimate of $E(B-V)$ is in the value of $\tau_{9.7}$, which will be refined as further observations in the $8-13 \mu \mathrm{m}$ window are obtained.

The profile of the interstellar silicate feature is (like the $A_{\mathrm{v}} / \tau_{9.7}$ relationship) difficult to determine and the " $\mu$ Cep" profile (Roche \& Aitken 1984) is usually taken as the standard (e.g. Bowey et al. 1998; Mathis 1998); this profile has been normalized at $\tau=0.105$ and $\lambda=9.7 \mu \mathrm{m}$ and included in Fig. $1 \mathrm{~b}$. The $\mu$ Cep profile seems consistent with our uncertainties in the determination of $\tau$, although the V4334 Sgr silicate profile may be somewhat narrower than the $\mu$ Cep feature; this may also be refined with further observation. The fitted $\mu$ Cep silicate feature has been superimposed on the linear continuum and the result compared with the data in Fig. 1a.

The various determinations of the reddening to V4334 Sgr are summarized in Table 2, from which it is evident that, with the exception of the early determination by Duerbeck \& Benetti (1996, but note that Kerber et al. (2000) obtained a much higher value using the same technique), the various methods lead to a remarkably consistent set of $E(B-V)$ values.

\section{Concluding remarks}

We have presented an 8-13 $\mu \mathrm{m}$ spectrum of V4334 Sgr, which shows the $9.7 \mu \mathrm{m}$ silicate feature in absorption. This is almost certainly interstellar in origin, which determines the reddening in the direction of V4334 Sgr to be $E(B-V)=0.66 \pm 0.12$.
In the case of V4334 Sgr, determinations of $E(B-V)$ that have depended on the visibility of the star (when it was visible) are unreliable because of the rapid changes that occur both in the star itself and in the degree of circumstellar reddening (e.g. Pavlenko \& Duerbeck 2001; Pavlenko \& Geballe 2002), while values based on observations of the PN are somewhat model-dependent. We note that the $E(B-V)$ reported here is based on the only method that uses the direct observation of the foreground material and as such must be one of the most secure.

Herwig (2001) has succeed in reproducing the rapid evolution of V4334 Sgr by assuming that the efficiency of element mixing in the convection zone in which the He flash occurs is smaller than expected on the basis of mixing length theory. His model requires a low $\left(\sim 0.6 M_{\odot}\right)$ mass and a distance close to $4 \mathrm{kpc}$. The reddening-distance relation in the direction of V4334 Sgr (Kimeswenger \& Kerber 1998) suggests a distance of $\sim 1.4 \mathrm{kpc}$ with the $E(B-V)$ deduced here, which will have implications for the modelling. While we caution against any revision of evolutionary theories until a more refined $\tau_{9.7}$ is obtained, it may be that we have not yet seen the last word on understanding the rate at which Sakurai's object is evolving.

Acknowledgements. We thank Dr S. Kimeswenger for his comments on an earlier version of this paper. The United Kingdom Infrared Telescope is operated by the Joint Astronomy Centre on behalf of Particle Physics and Astronomy Research Council (PPARC). We are grateful to S. K. Leggett and O. Kuhn for obtaining the spectrum of Sakurai's object after poor weather two nights earlier prevented us from doing so. TRG is supported by the Gemini Observatory, which is operated by the Association of Universities for Research in Astronomy, Inc., on behalf of the international Gemini partnership of Argentina, Australia, Brazil, Canada, Chile, the UK and the USA. VHT is supported by Keele University.

\section{References}

Asplund, M., Gustafsson, B., Lambert, D. L., \& Kameswara Rao, N. 1997, A\&A, 312, L17

Bowey, J. E., Adamson, A. J., \& Whittet, D. C. B. 1998, MNRAS, 298, 131

Duerbeck, H. W., \& Benetti, S. 1996, ApJ, 468, L111

Evans, A., \& Smalley, B. 2002, Sakurai's Object: What have we learned in the first five years?, Ap\&SS, 279, 1

Eyres, S. P. S., Richards, A. M. S., Evans, A., \& Bode, M. F. 1998, MNRAS, 297, 905 
Glasse, A. C., Atad-Ettedgui, E. I., \& Harris, J. W. 1997, in Proc. SPIE Vol. 2871, Optical Telescopes of Today and Tomorrow, ed. A. L. Ardeberg, 1197

Herwig, F. 2001, ApJ, 554, L71

Ivezić, Ž, \& Elitzur, M. 1997, MNRAS, 287, 799

Kerber, F., Palsa, R., Köppen, J., Blöcker, T., \& Rosa, M. R. 2000, ESO Messenger, 101, 27

Kerber, F., Pirzkel, N., \& Rosa, M. R. 2002, IAUC, 7879

Kimeswenger, S. 2002, Ap\&SS, 279, 79

Kimeswenger, S., \& Kerber, F. 1998, A\&A, 330, L41

Lynch, D. K., Rudy, R. J., Russell, R. W., et al. 2002, Ap\&SS, 279, 57

Martin, P. G., \& Whittet, D. C. B. 1990, ApJ, 357, 113

Mathis, J. S. 1998, ApJ, 497, 824
Molster, F. J., Yamamura, I., Waters, L. B. F. M., et al. 2001, A\&A, 366, 923

Pavlenko, Ya. V., \& Duerbeck, H. W. 2001, A\&A, 367, 933

Pavlenko, Ya. V., \& Geballe, T. R. 2002, A\&A, 390, 621

Pavlenko, Ya. V., Yakovina, L. A., \& Duerbeck, H. W. 2000, A\&A, 354,229

Pirzkal, N., Kerber, F., Clayton, G. C., de Marco, O., \& Rosa, M. R. 2001, Amer. Astron. Soc. Meet. 199, Abstract \#136.04

Pollacco, D. 1999, MNRAS, 304, 127

Roche, P. F., \& Aitken, D. K. 1984, MNRAS, 208, 481

Tyne, V. H., Evans, A., Geballe, T. R., et al. 2002, MNRAS, 334, 875

Yamamura, I., Dominik, C., de Jong, T., Waters, L. B. F. M., \& Molster, F. J. 2000, A\&A, 363, 629 